\title{
Surgical options for hip OA: digging beneath the surface of implant survival
}

\begin{abstract}
A $\mathrm{n}$ analysis of data from the National Joint Registry of England and Wales published in The Lancet has found that the implant survivorship for metal-on-metal hip resurfacing relates to sex of the patient and size of the device implanted. The authors report that the failure rate of hip resurfacing in female patients is "unacceptably high", and that the decision to undertake the procedure in men should be made on the basis of femoral head size as smaller head sizes are associated with greater risk of implant failure.
\end{abstract}

Hip resurfacing arthroplasty is often recommended as an alternative to total hip replacement (THR) for younger patients with hip osteoarthritis (OA) as it is bone-preserving and may be easier to revise than THR. Indeed, use of resurfacing procedures has increased rapidly, especially in young active patients as this group are known to have a higher failure rate of THR owing to implant wear. Nonetheless, some concerns have arisen about the procedure. "Hip resurfacing is a newer technology and recent reports have highlighted new mechanisms of failure such as localised adverse reactions to the metals and femoral neck fractures," says Ashley Blom of University of Bristol, who led the study. "Our National Joint Registry data, as well as the Australian Registry data, suggested higher than predicted failure rates. We thus wanted to quantify the risks of revision."

Using the England and Wales registry data, the researchers compared rates of revision surgery following 434,560 primary THRs performed between 2003 and 2011 , of which $7.4 \%(n=31,932)$ were resurfacing procedures. The mean age of patients who underwent hip resurfacing was 54 years, and more than two-thirds of these procedures were undertaken in men. Revision rates of resurfacings (femoral head sizes from $42 \mathrm{~mm}$ to $54 \mathrm{~mm}$ ) were compared with those of conventional stemmed THRs of different bearing surfaces: uncemented ceramic-on-ceramic ( $n=57,748$; head size $28 \mathrm{~mm}, 32 \mathrm{~mm}$ or $36 \mathrm{~mm}$ ) or metalon-polyethylene $(n=226,165 ; 28 \mathrm{~mm}$ head size with cemented, hybrid or uncemented fixations).

The analysis used flexible parametric survival models that estimate the cumulative incidence of revision in the presence of the competing risk of death. "Standard survival models treat death as censored information and thus can overestimate revision rates," says Blom. "A second advantage is that our models give an absolute rather than a relative risk."

The results of the analysis showed that risk of revision surgery following resurfacing increases with decreasing femoral head size in both men and women. Predicted 5-year revision rates for 55 -year-old men were $4.05 \%$ for a $46 \mathrm{~mm}$ head size, compared with $2.60 \%$ for a $54 \mathrm{~mm}$ head size; in 55-year-old women, $42 \mathrm{~mm}$ and $46 \mathrm{~mm}$ head sizes were associated with predicted 5-year revision rates of $8.33 \%$ and $6.07 \%$, respectively.

In men with larger femoral head sizes ( $\geq 54 \mathrm{~mm}$ ), revision rates were comparable with resurfacing and the alternative THR prostheses. However, these head sizes were used in only $23 \%$ of procedures in men in the registry data.

In women, implant survival was considerably lower for resurfacing than for the alternative stemmed THR surgical options analysed, even with larger femoral head sizes. Predicted 5-year revision rates for 55 -year-old women were $2.46 \%$ for $36 \mathrm{~mm}$ uncemented ceramic-on-ceramic THR and $1.47 \%$ for $28 \mathrm{~mm}$ cemented metal-on-polyethylene THR, compared with $6.07 \%$ for a $46 \mathrm{~mm}$ resurfacing procedure. On the basis of the results, the authors recommend that "resurfacing is not undertaken in women".

Given that the unit of analysis in the paper is the implant rather than the patient, however, some factors potentially important to the decision-making of orthopaedic surgeons treating patients with hip OA are not taken into account. Justin Cobb of Imperial College London, who was not involved with the study, points out that "The results reported ignore both the upside of hip resurfacing arthroplasty_being able to walk fast or even run, for instance, which is not possible after THR-and the downside of THR, which includes higher rates of both death and dislocation compared with hip resurfacing."

In addition, says Cobb, results for cemented THRs with larger diameter heads, such as the increasingly used $32 \mathrm{~mm}$ and $36 \mathrm{~mm}$ metal-on-polyethylene bearings, are not reported in the analysis, even though they "may fare rather worse in the registry".

In terms of implant survival (as measured by revision rate), the results of the analysis of data from the National Joint Registry of England and Wales show that metal-on-metal hip resurfacing arthroplasty has no advantage over conventional stemmed THRs for most patients, but the resurfacing procedure could nonetheless be suitable for some individuals.

Sarah Onuora 